Boise State University

ScholarWorks

8-15-2013

\title{
Fluctuant Magnetism in Metal Oxide Nanocrystals Capped with Surfactants
}

Aaron Thurber

Boise State University

Michael S. Jones

Boise State University

Dmitri Tenne

Boise State University

Charles B. Hanna

Boise State University

Alex Punnoose

Boise State University 
This is an author-produced, peer-reviewed version of this article. The final, definitive version of this document can be found online at Physical Review B: Condensed Matter and Materials Physics, published by American Physical Society. Copyright restrictions may apply. DOI: 10.1103/PhysRevB.88.085437.

\title{
Fluctuant Magnetism in Metal Oxide Nanocrystals Capped with Surfactants
}

\author{
Jianhui Zhang* \\ National Laboratory of Solid State Microstructures \\ Department of Physics \\ Nanjing University \\ Nanjing 210093 (China) \\ Shijie Xiong \\ National Laboratory of Solid State Microstructures \\ Department of Physics \\ Nanjing University \\ Nanjing 210093 (China) \\ Xinglong $\mathrm{Wu}^{*}$ \\ National Laboratory of Solid State Microstructures \\ Department of Physics \\ Nanjing University \\ Nanjing 210093 (China) \\ Aaron Thurber \\ Department of Physics \\ Boise State University \\ Boise, ID 83725 (USA) \\ Michael Jones \\ Department of Physics \\ Boise State University \\ Boise, ID 83725 (USA)

\section{Min Gu} \\ National Laboratory of Solid State Microstructures \\ Department of Physics \\ Nanjing University \\ Nanjing 210093 (China)
}

\author{
Zhongda Pan \\ National Laboratory of Solid State Microstructures \\ Department of Physics \\ Nanjing University \\ Nanjing 210093 (China) \\ Dmitri A. Tenne \\ Department of Physics \\ Boise State University \\ Boise, ID 83725 (USA) \\ Charles B. Hanna \\ Department of Physics \\ Boise State University \\ Boise, ID 83725 (USA) \\ Youwei Du \\ National Laboratory of Solid State Microstructures \\ Department of Physics \\ Nanjing University \\ Nanjing 210093 (China)
}

\author{
Alex Punnoose* \\ Department of Physics \\ Boise State University \\ Boise, ID 83725 (USA)
}

\begin{abstract}
We demonstrate experimentally that magnetism in $\mathrm{ZnO}, \mathrm{TiO}_{2}, \mathrm{CeO}_{2}$, and $\mathrm{SnO}_{2}$ nanocrystals (NCs) has a fluctuant nature that varying with capping surfactant type and concentration. By developing a forced hydrolysis approach with additional post-processing for the synthesis and surfactant capping of these NCs, we effectively avoid the influence of size, shape, and magnetic impurities on the magnetic behavior of NCs, thus revealing the systematic influence of the capping surfactants on the NC magnetism. The x-ray photoelectron spectroscopy results and theoretical calculations clearly show that the magnetism fluctuation with surfactant concentration can be attributed to the periodic variation of spins, which arises from the concentration dependent electron transfer from surfactants to NCs. Our results not only explain the previously reported seemingly irregular magnetism induced by capping surfactants, but also provide an effective approach to tune or optimize the NC magnetism.
\end{abstract}


This is an author-produced, peer-reviewed version of this article. The final, definitive version of this document can be found online at Physical Review B: Condensed Matter and Materials Physics, published by American Physical Society. Copyright restrictions may apply. DOI: $10.1103 /$ PhysRevB.88.085437.

PACS number(s): 75.75.Cd, 81.40.Rs, 75.70.Rf

*Authors to whom correspondence should be addressed; electronic mail:

zhangjh@nju.edu.cn,hkxlwu@nju.edu.cn, and apunnoos@boisestate.edu

\section{INTRODUCTION}

Developing ferromagnetism in semiconducting metal oxide nanocrystals (NCs) has been intensively investigated due to their potential applications in several areas such as spintronics, information storage, and biomedicine. ${ }^{1-14}$ Ferromagnetism (FM) can be induced in non-magnetic metal oxide NCs by a variety of methods or factors. For example, intrinsic defects such as oxygen or cation vacancies in pure metal oxides (such as $\mathrm{ZnO}, \mathrm{CeO}_{2}$, or $\mathrm{SnO}_{2}$ ) can lead to room temperature (RT) FM. $\cdot^{2-5}$ Defects intentionally induced by doping nonmagnetic elements (such as Ti and V) into metal oxides can also produce RT FM. ${ }^{6}$ A more traditional method is doping magnetic impurities (such as $\mathrm{Co}, \mathrm{Ni}, \mathrm{Mn}$, and $\mathrm{Cr}$ ) to introduce magnetic moments directly. ${ }^{7-9}$ Recently, it was found that capping metal oxides with inorganic (such as $\mathrm{NH}_{3}{ }^{10}$ ) or organic (such as dedecanethiol, $\mathrm{THIOL}^{11}$ ) molecules can effectively induce FM via changing electronic structure. This approach might be promising for synthesis of metal oxide semiconductor NCs with optimized FM because there are numerous molecules to be chosen for capping and modifying the oxide cores. Moreover, it can avoid the problem of clustering of magnetic elements. ${ }^{12}$ However, the influence of the capping surfactants on magnetism has been found to vary with surfactants, and show inconsistent results in some similar systems. For example, Gamelin et al. reported no RT FM in dodecylamine (AMINE)-capped ZnO NCs, ${ }^{13}$ whereas Jiang et al. observed FM in AMINE-capped $\mathrm{ZnO}$ NCs and revealed FM to arise from the capping AMINE. ${ }^{14}$ Furthermore, Garcia et al. found the saturation magnetization $\left(M_{s}\right)$ of undoped $\mathrm{ZnO}$ NCs to increase with the capping surfactants in the order of tryoctylphosphine oxide (TOPO) < AMINE $<$ THIOL. ${ }^{11}$

Although it is not completely clear how the capping molecules affect the magnetism of the metal oxide NCs, electron configuration variations induced by capping molecules have been considered to be a crucial factor. ${ }^{10,11,14}$ Besides the surfactant type, the electron configuration is also expected to vary with the capping surfactant concentration (SC). Namely, the capping SC might be able to change the NC magnetism, thus leading to the seemly irregular magnetism variations described above. However, until now, systematic studies of the influences of the capping SC on magnetism of metal oxide NCs have been very limited. It is possible that capping NCs with surfactants is often accompanied with size and shape variations of NCs and defect introduction, and therefore only a combined effect is observed. ${ }^{13,15,16}$ To reveal how the capping surfactants affect the magnetism of the oxide NCs, a forced hydrolysis approach with additional post-processing for the synthesis and surfactant capping of $\mathrm{ZnO} \mathrm{TiO}_{2}$ $\mathrm{SnO}_{2}$, and $\mathrm{CeO}_{2} \mathrm{NCs}$ has been developed here. This ensures that the size and shape of NCs remain unchanged and only the systematic influence of the surfactants on the $\mathrm{NC}$ magnetism is investigated. Our results clearly show that the NC magnetism is intimately related to both the type and amount of capping surfactants, and can be tuned simply by modifying their capping SC.

\section{EXPERIMENTAL CONDITIONS}

All the analytical reagent grade chemicals were used as received. For the synthesis of $\mathrm{ZnO}$ NCs, $400 \mathrm{~mL}$ of DEG containing $8 \mathrm{~g}$ of $\mathrm{Zn}(\mathrm{Ac})_{2} \cdot 2 \mathrm{H}_{2} \mathrm{O}$ was heated to $180^{\circ} \mathrm{C}$ under stirring for $30 \mathrm{~min}$. The resulting NCs were washed with ethanol 3 times via centrifugation and sonication. For the syntheses of $\mathrm{TiO}_{2}, \mathrm{SnO}_{2}, \mathrm{CeO}_{2} \mathrm{NCs}$, nanopure water (4 $\mathrm{mL}$ for $\mathrm{TiO}_{2}, 8 \mathrm{~mL}$ for $\mathrm{SnO}_{2}$ and $\left.\mathrm{CeO}_{2}\right)$ was added to $\mathrm{DEG}(400 \mathrm{~mL})$ containing $8 \mathrm{~mL}$ of Ti( $\left(\mathrm{OCH}\left(\mathrm{CH}_{3}\right)_{2}\right)_{4}$ for $\mathrm{TiO}_{2}$, or $8 \mathrm{~g}$ of $\mathrm{Sn}(\mathrm{Ac})_{4}\left(\right.$ for $\left.\mathrm{SnO}_{2}\right)$ or $\mathrm{Ce}(\mathrm{Ac})_{3} \cdot 1.5 \mathrm{H}_{2} \mathrm{O}\left(\right.$ for $\left.\mathrm{CeO}_{2}\right)$ at $80{ }^{\circ} \mathrm{C}$, and then the system was heated at $180^{\circ} \mathrm{C}$ for 1 $\mathrm{h}$. The resulting product was also washed with ethanol 3 times via centrifugation and sonication. The as-prepared product was dried at $50^{\circ} \mathrm{C}$ to obtain a powder sample, or dispersed in nanopure water $(80 \mathrm{~mL})$ for post-treating with surfactants. $\mathrm{CeO}_{2} \mathrm{NCs}$ were dried at $80^{\circ} \mathrm{C}$ for $12 \mathrm{~h}$ before the surfactant capping.

To ensure that all the NCs capped with different surfactants are identical in their initial crystal quality, size and shape, thus ensuring that we are effectively studying only the influence of the surfactants on the NC magnetism, a surfactant post-treating method using the same NC source was developed. Briefly, the as-prepared ZnO NCs were dispersed in $80 \mathrm{~mL}$ of nanopure water to produce a stock suspension. An ethanol solution $(20 \mathrm{~mL})$ of surfactants $(75$ $\mathrm{mM}$ ) was used to suspend the $\mathrm{ZnO} \mathrm{NCs} \mathrm{that} \mathrm{were} \mathrm{fabricated} \mathrm{by} \mathrm{centrifuging} 10 \mathrm{~mL}$ of stock suspension. After 50 
This is an author-produced, peer-reviewed version of this article. The final, definitive version of this document can be found online at Physical Review B: Condensed Matter and Materials Physics, published by American Physical Society. Copyright restrictions may apply. DOI: 10.1103/PhysRevB.88.085437.

min of sonication, the product was washed with ethanol twice and dried at $50^{\circ} \mathrm{C}$ to obtain a powder sample. A small concentration $(5 \mathrm{mM})$ was used for polyvinylpyrrolidone (PVP) due to its large molecular weight $\left(M_{\mathrm{w}}=58,000\right)$ which makes it hard to prepare a high-concentration solution. An aqueous solution $(20 \mathrm{~mL})$ of $\mathrm{NaCl}(1 \mathrm{mM})$ and poly (acryl acid) (PAA, $M_{w}=1800,10 \mathrm{mg} / \mathrm{mL}$ ) followed by twice washing with water was used to cap PAA.

The product morphology was examined using a JEOL JEM-2100HR transmission electron microscope (TEM) with an accelerating potential of $200 \mathrm{kV}$. Cryststallographic phases of the samples were studied by x-ray diffraction (XRD) using a Philips X'pert MPD diffractometer with a $\mathrm{Cu} \mathrm{K}_{\alpha}$ X-ray source in Bragg-Brentano geometry. X-ray photoelectron spectroscopy (XPS) data were recorded by a Physical Electronics Versa probe using a raster-scanned microfocused monochromatic Al $\mathrm{K}_{\alpha}$ X-ray $(1486.7 \mathrm{eV})$ source. High resolution XPS spectra were obtained with a pass energy of $23.5 \mathrm{eV}$ and at a take-off angle of $45^{\circ}$. The trace magnetic impurity in samples was detected by an inductively coupled plasma mass spectrometry (ICP-MS, PQEXCe II). The magnetization hysteresis of the dried powder samples was acquired at RT using a vibrating sample magnetometer. The noise floor of the VSM magnetometer is 0.1 microemu for a collection time of $1 \mathrm{~second} /$ point and 0.75 microemu for a collection time of $0.1 \mathrm{~s} / \mathrm{pt}$, which ensures that the weak magnetic signal down to 1 microemu in our samples can be precisely measured.

\section{RESULTS AND DISCUSSION}

The TEM images disclose that both ZnO NCs post-treated with TOPO (Fig 1a) and PAA (Fig. 1b) show short nanorod-like shapes with an average diameter of $\sim 9 \mathrm{~nm}$ and length of $\sim 15 \mathrm{~nm}$. The corresponding XRD patterns (Fig. 1c) indicate that all the $\mathrm{ZnO}$ NCs post-treated with different surfactants have the same pure wurtzite $\mathrm{ZnO}$ phase and a similar average size $(\sim 9.2 \mathrm{~nm}$, obtained using Scherrer formula), which agrees well with the TEM observations. The results show that the capping surfactants do not affect the $\mathrm{ZnO} \mathrm{NC}$ size and shape, and therefore these samples really allow a systematic and effective study of the capping-surfactant influence on the $\mathrm{ZnO} \mathrm{NC}$ magnetism. As seen in Fig. 1d, all the ZnO NCs treated with different surfactants show a weak FM, which does not arise from the impurity in surfactants or the measurement artifacts because all the pure surfactants used here show their diamagnetic nature under the same measurement conditions (Fig. S1) ${ }^{17}$ The NC $M_{s}$ increases with capping surfactants in the order of THIOL $\leqq$ PAA $\leqq$ PVP $<$ AMINE $<$ CTAB $<$ TOPO, which is different from the results reported earlier. ${ }^{11}$ As addressed below, this may be ascribed to the higher SC used here to cap NCs, because the capping SC can greatly affect the NC magnetism.

TOPO, PVP, and PAA were selected for a systematic study of the influence of capping SC on the ZnO NC magnetism because capping by these molecules leads to the maximum or minimum $M_{s}$. The high resolution XPS results clearly show that the actual surfactant amount deposited onto $\mathrm{ZnO}$ NCs is proportional to the capping SC. As seen in Table SI ${ }^{17}$ with increasing SC, the number of surfactant molecules adsorbed onto per ZnO NC increases, but tends to be saturated at high SC, which may be ascribed to the reduction of the surface $\mathrm{Zn}$ atoms of NCs available for the surfactant bonding, and the steric hindrance effect among surfactant molecules. According to the calculation results based on the NC surface area, surfactant molecule size, and the adsorbed surfactant molecule number per NC, it is deduced that only a monolayer of surfactant molecules is formed on the $\mathrm{ZnO}$ NCs after treating with all the surfactants. As shown in Fig. 2a, with increasing SC, both TOPO and PVP capping initially increases the saturation magnetization $M_{s}$ of $\mathrm{ZnO} \mathrm{NC}$ abruptly by a factor of about 7, followed by a dramatic decrease of $M_{s}$. With further increasing $\mathrm{SC}$, the $M_{s}$ variation trend repeats itself, but at a much lower frequency. The $\mathrm{ZnO} \mathrm{NC}$ magnetism also varies with the capping-PAA concentration in a similar manner, but its variations induced by the capping of high concentrations of PAA (which will apparently dissolve and change the $\mathrm{ZnO} \mathrm{NCs}$ ) have not been investigated here. As shown in Tables SI-II, ${ }^{17}$ the maximum adsorbed surfactant/ZnO weight ratio and magnetic impurity (including $\mathrm{Cr}, \mathrm{Mn}, \mathrm{Fe}, \mathrm{Co}$, and $\mathrm{Ni}$ ) concentration in $\mathrm{NCs}$ are below $3.5 \%$ and $3.5 \mathrm{ppb}$, respectively. Therefore, the influence of the small difference in the adsorbed-surfactant weight and the negligible amount of magnetic impurities on the above large FM variations can be excluded here.

To check whether the above magnetism variation with capping SC is a general trend among metal oxide NCs, we also investigated the $\mathrm{SC}$ influences of TOPO and PAA on the magnetism of $\mathrm{SnO}_{2}, \mathrm{TiO}_{2}$, and $\mathrm{CeO}_{2} \mathrm{NCs}$ made in a similar way as the $\mathrm{ZnO}$ NCs. TEM and XRD results (Figs. S2-4) ${ }^{17}$ show that the as-prepared $\mathrm{SnO}_{2}, \mathrm{TiO}_{2}$, and $\mathrm{CeO}_{2}$ NCs have the short nanorod shapes with an average diameter of $\sim 4 \mathrm{~nm}$, and high-purity tetragonal rutile, anatase, and fluorite structures, respectively. The capping of both TOPO and PAA has no detectable influences on their crystalline phase, size, and shape, thus excluding the effect of factors other than the concentration of the capping surfactant on the $\mathrm{NC}$ magnetism. As expected, $\mathrm{FM}$ in $\mathrm{SnO}_{2}, \mathrm{TiO}_{2}$, and $\mathrm{CeO}_{2} \mathrm{NCs}$ is also greatly enhanced after the 
initial capping of TOPO and PAA, and then fluctuates with increasing SC as illustrated in Fig. 2b-c and Fig. 3. Like in the case of $\mathrm{ZnO} \mathrm{NCs}$, the undoped and uncapped $\mathrm{SnO}_{2}, \mathrm{TiO}_{2}$, and $\mathrm{CeO}_{2} \mathrm{NCs}$ also show very weak FM after subtracting the diamagnetic background, which might arise from intrinsic defects such as oxygen vacancies. ${ }^{2,3,5}$ The strongest FM $\left(M_{s}=8.7 \mathrm{memu} \mathrm{g}^{-1}\right)$ is observed in $\mathrm{SnO}_{2} \mathrm{NCs}$ treated with $40 \mathrm{mg} \mathrm{mL}^{-1}$ of TOPO, while the largest FM enhancement ( $\sim 40$ fold) is found in $\mathrm{TiO}_{2} \mathrm{NCs}$ treated with $10 \mathrm{mg} \mathrm{mL}^{-1}$ of PAA.

The above magnetism fluctuation with SC can be ascribed to an alteration of electronic configuration ${ }^{10,13,14}$ induced by the electron transfer between NCs and surfactants, ${ }^{18-20}$ which is revealed by the XPS spectra. The typical XPS spectra of $\mathrm{ZnO}$ and $\mathrm{CeO}_{2} \mathrm{NCs}$ before and after post-treating with TOPO, PVP, and PAA are shown in Fig. 4, and Fig. S5, ${ }^{17}$ respectively. Treating ZnO NCs with TOPO, PVP, or PAA, apparently shifts the $\mathrm{Zn} 2 \mathrm{p}_{3 / 2}$ XPS peak to a low binding energy (Fig. 4), indicating that $\mathrm{ZnO}$ receives electrons from the surfactants. ${ }^{20}$ Similarly, the TOPO and PAA molecules that are linked to the $\mathrm{CeO}_{2} \mathrm{NCs}$ also donate electrons, and this leads to the appearance of $\mathrm{Ce}^{3+} \mathrm{XPS}$ peaks ${ }^{21}$ (Fig. S5). As shown in Fig. 4, with the increase of the capping SC, the Zn $2 p_{3 / 2}$ XPS peak of ZnO NCs continuously shifts to a lower binding energy, but the relative shift gradually slows down. This is consistent with the expected variations in the number of the adsorbed surfactant molecules per $\mathrm{ZnO} \mathrm{NC}$ as SC increases. The relationship between the electron transfer and the NC magnetism is clearly demonstrated in Fig. 5, showing the $\mathrm{ZnO}$ $\mathrm{NC} M_{s}$ plotted as a function of the Zn 2 $\mathrm{p}_{3 / 2}$ XPS peak shift induced by the capping of TOPO, PVP, and PAA at different concentrations. As shown in the figure, with the increase of the XPS peak shift to lower binding energ, (i.e., the number of electrons transferred), the NC $M_{s}$ initially increases to a maximum followed by decreasing to a minimum, and then repeats the variation trend as the XPS peak shift increases further. These results clearly reveal the fluctuation nature of the NC magnetism with the number of the extra electrons donated by the capping surfactants. We also noted that the fluctuation rate of the NC magnetism with the SC or the number of electrons transferred varies with surfactants. This can be attributed to the variation in the interaction strength or bonding structure between NCs and surfactants, depending on the capping molecules. As shown in Figs. 4-5, compared with the functional $\mathrm{O}$ atoms of PAA, the functional $\mathrm{P}$ or $\mathrm{N}$ atoms of TOPO or PVP have relatively lower electronegativity and donate electrons to NCs more easily, thus leading to a larger Zn 2 $\mathrm{p}_{3 / 2}$ XPS peak shift towards low binding energies. The different NC-surfactant interactions should affect the kinetic energy as well as the interaction or coupling among the extra surface electrons donated by the capping surfactants, ${ }^{18,19}$ thus lead to the different fluctuation rates of the NC magnetism with SC.

To explain the abovementioned fluctuation of the magnetism of NCs with SC theoretically, we took a ZnO NC with an adsorbed TOPO molecule as an example to carry out the first-principle calculations and structure optimization. The first-principle calculations were performed using the generalized gradient approximation (GGA) of the Perdew, Burke, and Ernzerholf (PBE) form ${ }^{22}$ under package CASTEP, ${ }^{23}$ in which a plane-wave norm-conserving pseudopotential method ${ }^{24}$ was used. We used a kinetic energy cutoff of $400 \mathrm{eV}$ to represent the single-particle wave functions. The NC had a diameter of $9.5 \AA$, and adsorbed a TOPO molecule. To form a periodic structure for the calculation with CASTEP package, the unit cells of size $25 \times 20 \times 16 \AA^{3}$, each of which containing a NC, a TOPO molecule, and vacuum space, were periodically placed along three directions. The geometry of the configuration was optimized using BFGS minimizer in the CASTEP package with default convergence tolerances: $2 \times 10^{-5} \mathrm{eV}$ for energy change, $0.05 \mathrm{eV} / \AA$ for maximum force, and $0.002 \AA$ for maximum displacement. ${ }^{25}$ Fig. $6 \mathrm{a}$ shows the optimized structure of the $\mathrm{NC}$ with an adsorbed TOPO molecule and the spatial distribution of electron density. It can be seen that with increase in the NC-TOPO interaction, several oxygen atoms in the NC are pulled towards the TOPO molecule. These oxygen atoms carry a dense electron cloud due to the charge transfer from the TOPO molecule. By assigning the charge to every atom, we found the average charge of an oxygen atom in the NC to be $-0.787 e$ ( $\mathrm{e}$ is the elementary charge), while that of a zinc atom was only $0.657 e$. So, even though there are $24 \mathrm{Zn}$ atoms and only $21 \mathrm{O}$ atoms in the $\mathrm{NC}$, the total charge in the $\mathrm{NC}$ is still negative, $-0.759 e$, indicating the net electron transfer from TOPO molecule to NC. This is consistent with our experimental result shown in Fig. 4a, where the shift of the $\mathrm{Zn} 2 \mathrm{p}_{3 / 2}$ XPS peak towards low binding energies induced by the TOPO capping indicates the transfer of electrons to $\mathrm{ZnO} \mathrm{NCs}$ from TOPO.

It was shown theoretically that the total orbital momentum of the Fermi level in non-magnetic NCs can oscillate with increasing the number of extra electrons donated by the capping surfactant, with a maximum occurring at one-half occupation. ${ }^{18,19}$ The above magnetism fluctuation of NCs with SC may arise from the variations of the number of electrons transferred to NCs from the capping surfactants. To confirm this, we performed the first-principle calculations of the spins in NCs with different numbers of extra electrons. At this stage we did not include the surfactant molecules so that we could deal with larger NC clusters, $14 \AA$ in diameter. The effect of 
This is an author-produced, peer-reviewed version of this article. The final, definitive version of this document can be found online at Physical Review B: Condensed Matter and Materials Physics, published by American Physical Society. Copyright restrictions may apply. DOI: 10.1103/PhysRevB.88.085437.

electron transfer between NCs and surfactant molecules was simulated by setting different charges of NCs. In Fig. 6b-f, we plot the integrated densities $I(E)$ of spins for NCs with different charges $\mathrm{Z}$, where $I(E)$ is defined as $I(E)=\int_{-\infty}^{E} d \varepsilon\left[\rho_{\uparrow}(\varepsilon)-\rho_{\downarrow}(\varepsilon)\right]$ with $\rho_{\uparrow}(\varepsilon)$ and $\rho_{\downarrow}(\varepsilon)$ being the partial densities of states of spin up and down electrons in the $\mathrm{NC}$, respectively. So $I(E)$ corresponds to the total spin of electrons with energies below $E$ in the NC. The value of $I(E)$ at the Fermi energy $E=0$ is just the total spin of electrons as the states with energies below zero are occupied. Fig. $6 \mathrm{~g}$ shows the variation of $I(E=0)$ with charge number. As expected, the charge variation in NCs not only shifts the Fermi level, but also largely alters the density of spin states. With increasing the charge from zero, the NC spin becomes nonzero and the magnitude oscillates. These calculation results explain the general trend of the measured $\mathrm{NC}$ magnetization variations with $\mathrm{SC}$.

\section{CONCLUSIONS}

In summary, we have developed a forced hydrolysis approach with additional post-processing to synthesize and coat uniform and size-homogeneous $\mathrm{ZnO}, \mathrm{TiO}_{2}, \mathrm{CeO}_{2}$, and $\mathrm{SnO}_{2} \mathrm{NCs}$ with different types and concentrations of surfactants in order to investigate the systematic influence of the capping-surfactant on NC magnetism. As confirmed by the TEM, XRD, XPS, and ICP-MS results, the influence of the NC size and shape, and any role of magnetic impurities such as $\mathrm{Cr}, \mathrm{Mn}, \mathrm{Fe}, \mathrm{Co}$, and $\mathrm{Ni}$ on the magnetic properties of NCs have been effectively ruled out. Thus, the magnetism fluctuation with the capping-surfactant type and/or concentration in these NCs has been demonstrated. The XPS results clearly show that the amount of electron transfer from the capping surfactants to NCs is dependent on the SC-, and this causes the NC magnetism to fluctuate. The theoretical calculations confirm the electron transfer from the capping molecules to NCs, and clearly show that the electron transfer to NCs can lead to a periodic variation of spins, thus the observed NC magnetism fluctuation. Our results reasonably explain the seemingly irregular magnetism induced by capping surfactants in previous reports, and provide an effective approach to tune or optimize the NC magnetism. Compared with modifying the NC magnetism by introducing defects and/or dopants, which depends on the preparation conditions and procedures, we find that varying the surfactant type/concentration via post-processing to be a simple and highly reproducible method of tuning the NC magnetism.

\section{ACKNOWLEDGMENTS}

This work was supported by National Basic Research Programs of China (Nos. 2012CB932304, 2011CB922102, 2011CB933400, and 2012CB93400), program for new Century Excellent Talents at Nanjing University, and project (No. 61264008) of NSFC. Partial support was also from NSF EAGER DMR-1137419, ARO W911NF-09-1-0051, NSF CBET 1134468, and NSF DMR-0906618 grants at Boise State University. 


\section{References}

${ }^{1}$ S. Z. Deng, H. M. Fan, M. Wang, M. R. Zheng, J. B. Yi, R. Q. Wu, H. R. Tan, C. H. Sow, J. Ding, Y. P. Feng, K. P. Loh, ACS Nano 4, 495 (2010).

${ }^{2}$ D. Sanyal, M. Chakrabarti, T. K. Roy, A. Chakrabarti, Phys. Lett. A 371, 482 (2007).

${ }^{3}$ Q. Wang, Q. Sun, G. Chen, Y. Kawazoe, P. Jena, Phys. Rev. B 77, 205411 (2008).

${ }^{4}$ S. Y. Chen, Y. H. Lu, T. W. Huang, D. C. Yan, C. L. Dong, J. Phys. Chem. C 114, 19576 (2010).

${ }^{5}$ L. Zhang, S. Ge, Y. Zuo, B. Zhang, L. Xi, J. Phys. Chem. C 114, 7541 (2010).

${ }^{6}$ M. Venkatesan, C. B. Fitzgerald, J. M. D. Coey, Nature 430, 630 (2004).

${ }^{7}$ O. D. Jayakumar, C. Sudakar, A. Vinu, A. Asthana, A. K. Tyagi, J. Phys. Chem. C 113, 4814 (2009).

${ }^{8}$ S. B. Ogal, Adv. Mater. 22, 3125 (2010).

${ }^{9}$ L. Bizo, M. Allix, H. Niu, M. J. Rosseinsky, Adv. Funct. Mater. 18, 777 (2008).

${ }^{10}$ E.-Z. Liu, J. Z. Jiang, J. Phys. Chem. C 113, 16116 (2009).

${ }^{11}$ M. A. Garcia, J. M. Merino, E. F. Pinel, A. Quesada, J. de la Venta, M. L. R. González, G. R. Castro, P. Crespo, J. Llopis, J. M. González-Calbet, A. Hernando, Nano Lett. 7, 1489 (2007).

${ }^{12}$ S.-P. Huang, H. Xu, I. Bello, R.-Q. Zhang, Chem. Eur. J. 16, 13072 (2010).

${ }^{13}$ K. R. Kittilstved, D. R. Gamelin, J. Am. Chem. Soc. 127, 5292 (2005).

${ }^{14}$ J. F. Liu, E.-Z. Liu, H. Wang, N. H. Su, J. Qi, J. Z. Jiang, Nanotechnology 20,165702 (2009).

${ }^{15}$ A. P.Thurber, G. L. Beausoleil, G. A. Alanko, J. J. Anghel, M. S. Jones, L. M. Johnson, J. Zhang, C. B. Hanna, D. A. Tenne, A. Punnoose, J. Appl. Phys. 109, 07C305 (2011).

${ }^{16}$ L. Guo, S. Yang, C. Yang, P. Yu, J. Wang, W. Ge, G. K. L. Wong, Appl. Phys. Lett. 76, 2901 (2000).

${ }^{17}$ See supplementary material at [URL will be inserted by publisher] for Fig. S1 (hysteresis loops of pure CS), Figs. S2-4 (TEM and XRD results of $\mathrm{SnO}_{2}, \mathrm{TiO}_{2}$, and $\mathrm{CeO}_{2} \mathrm{NCs}$ before and after CS capping), Fig. S5 (XPS spectra of $\mathrm{CeO}_{2} \mathrm{NCs}$ treated by CS), Table SI (Carbon/Zn atomic ratio, adsorbed surfactant/ZnO weight ratio, and adsorbed surfactant molecule number per NC of ZnO NCs treated by CS), Table SII (ICP-MS results of ZnO CNs treated by CS).

${ }^{18}$ A. Hernando, P. Crespo, M. A. García, M. Coey, A. Ayuela, P. M. Echenique, Phys. Status Solidi B 248, 2352 (2011).

${ }^{19}$ A. Hernando, M. A. García, J. Nanopart Res. 13, 5595 (2011).

${ }^{20}$ J. J. Uhlrich, R. Franking, J. H. Robert, T. F. Kuech, J. Phys. Chem. C 113, 21147 (2009).

${ }^{21}$ M. A. Henderson, C. L. Perkins, M. H. Engelhard, S. Thevuthasan, C. H. F. Peden, Surf. Sci. 526,1 (2003).

${ }^{22}$ J. P. Perdew, K. Burke, M. Ernzerhof, Phys. Rev. Lett. 77, 3865 (1996).

${ }^{23}$ S. J. Clark, M. D. Segall, C. J. Pickard, P. J. Hasnip, M. J. Probert, K. Refson, M. C. Payne, Zeitschrift fuer kristallographie 220,567(2005).

${ }^{24}$ D. R. Hamann, M. Schluter, C. Chiang, Phys. Rev. Lett. 43, 1494 (1979).

${ }^{25}$ B. G. Pfrommer, M. Cote, S. G. Louie, M. L. Cohen, J. Comput. Phys. 131, 233 (1997). 
This is an author-produced, peer-reviewed version of this article. The final, definitive version of this document can be found online at Physical Review B: Condensed Matter and Materials Physics, published by American Physical Society. Copyright restrictions may apply.

DOI: 10.1103/PhysRevB.88.085437.

\section{Figure captions}

Color online FIG. 1. Typical TEM images of ZnO NCs post-treated with TOPO (a) and PAA (b); XRD patterns and hysteresis loops of ZnO NCs treated with different surfactants are shown in (c) and (d), respectively. The diamagnetic background of the measurement system including the plastic capsules used to pack samples in (d) was subtracted for all the curves, and $1 \mathrm{memu} \mathrm{g}^{-1}$ is defined as $10^{-3} \mathrm{emu} \mathrm{g}^{-1}$.

Color online FIG. 2. (a) $M_{s}$ from the $\mathrm{ZnO}$ NCs as function of the capping-surfactant concentration. (b) $M_{s}$ from the $\mathrm{SnO}_{2}, \mathrm{TiO}_{2}$, and $\mathrm{CeO}_{2} \mathrm{NCs}$ as functions of the capping-TOPO concentration. For clarity, the $M_{s}$ of $\mathrm{CeO} \mathrm{NCs}_{2}$ been multiplied by a factor of 20. (c) Typical hysteresis loops of $\mathrm{SnO}_{2}$ NPs treated by TOPO at different concentrations. The diamagnetic background was subtracted for all the curves.

Color online FIG. 3. Typical hysteresis loops of $\mathrm{SnO}_{2}$ (a), $\mathrm{TiO}_{2}$ (b), and $\mathrm{CeO}_{2}$ (c) $\mathrm{NPs}$ treated by PAA at different concentrations. The diamagnetic background was subtracted for all the curves. (d) $M_{s}$ as function of the capping-PAA concentration.

Color online FIG. 4. Zn $2 \mathrm{p}_{3 / 2}$ XPS spectra of the selected ZnO NC samples treated by TOPO (a), PVP (b), and PAA (c) with different concentrations. The binding energy scales have been charge-referenced using the $\mathrm{C} 1 \mathrm{~s}$ peak at $284.8 \mathrm{eV}$.

Color online FIG. 5. ZnO NC $M_{s}$ versus the Zn $2 \mathrm{p}_{3 / 2}$ XPS peak shift induced by the capping of TOPO, PVP, and PAA at different concentrations.

Color online FIG. 6. (a) Optimized structure and electron density distribution of a $\mathrm{ZnO} \mathrm{NC}$ with an adsorbed TOPO molecule, taken from the first-principle calculation. The gray-blue, red, violet, gray, and white balls represent $\mathrm{Zn}$, $\mathrm{O}$, $\mathrm{P}, \mathrm{C}$, and $\mathrm{H}$ atoms, respectively (for print in grayscale: the big black, small black, big grey, moderate size black, and small white grey balls represent $\mathrm{Zn}, \mathrm{O}, \mathrm{P}, \mathrm{C}$, and $\mathrm{H}$ atoms, respectively). (b)-(f) Integrated density of spin of a $\mathrm{ZnO}$ $\mathrm{NC}$ with different transferred charges $\mathrm{Z}$ given in the legend. The Fermi level is at the zero energy. (g) The variation of spin in the NC with transferred charge. 
This is an author-produced, peer-reviewed version of this article. The final, definitive version of this document can be found online at Physical Review B: Condensed Matter and Materials Physics, published by American Physical Society. Copyright restrictions may apply. DOI: 10.1103/PhysRevB.88.085437.
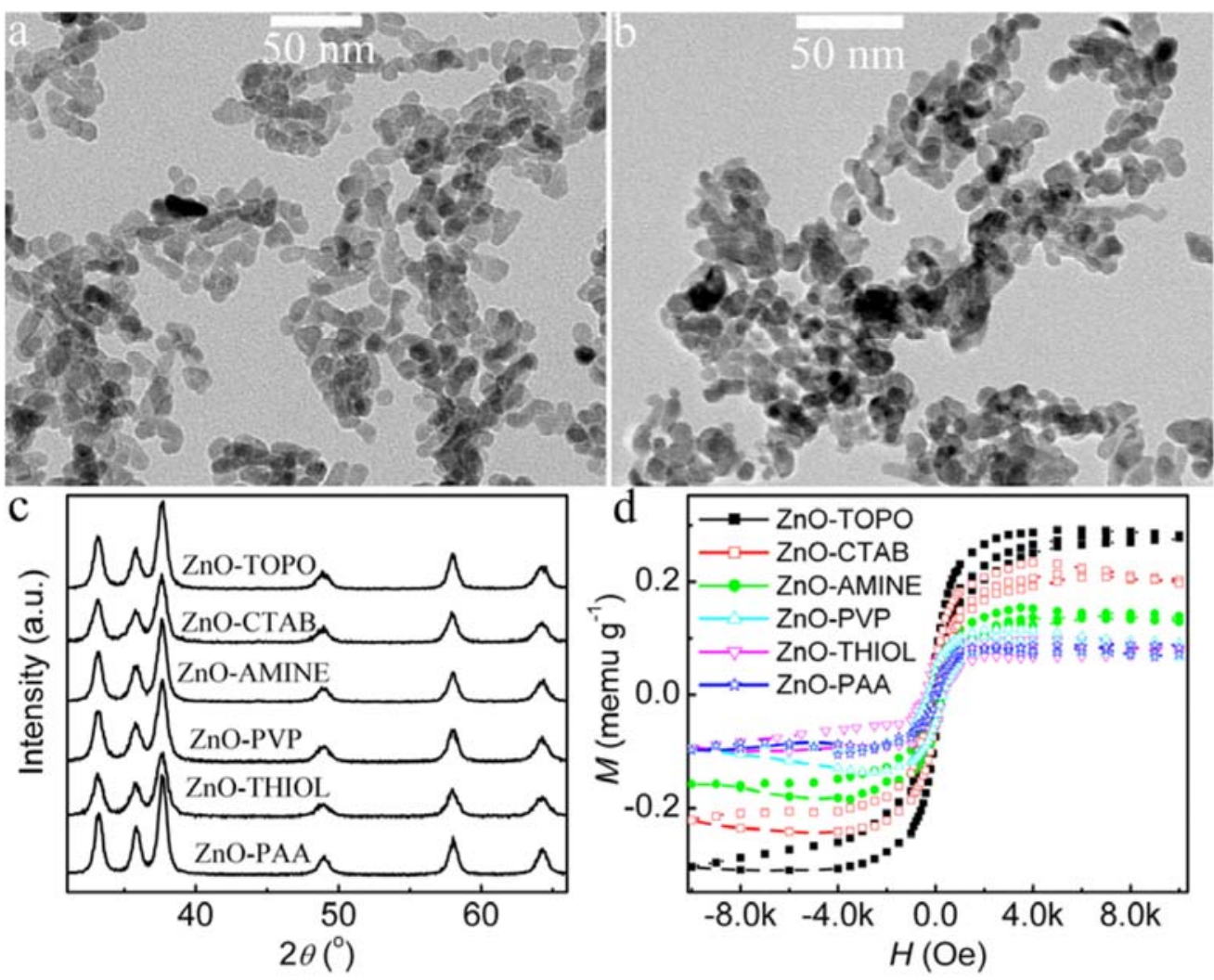

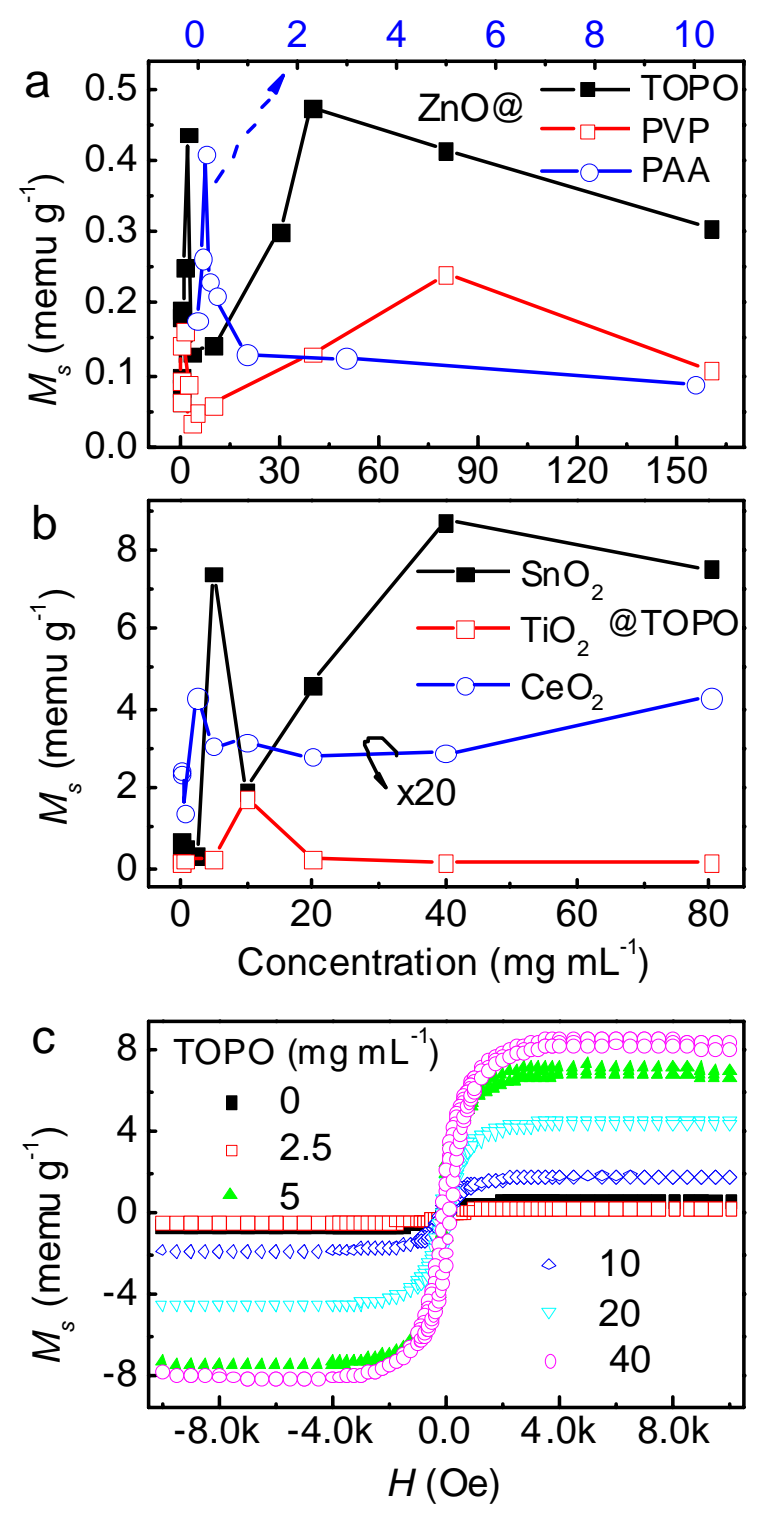
This is an author-produced, peer-reviewed version of this article. The final, definitive version of this document can be found online at Physical Review B: Condensed Matter and Materials Physics, published by American Physical Society. Copyright restrictions may apply. DOI: 10.1103/PhysRevB.88.085437.

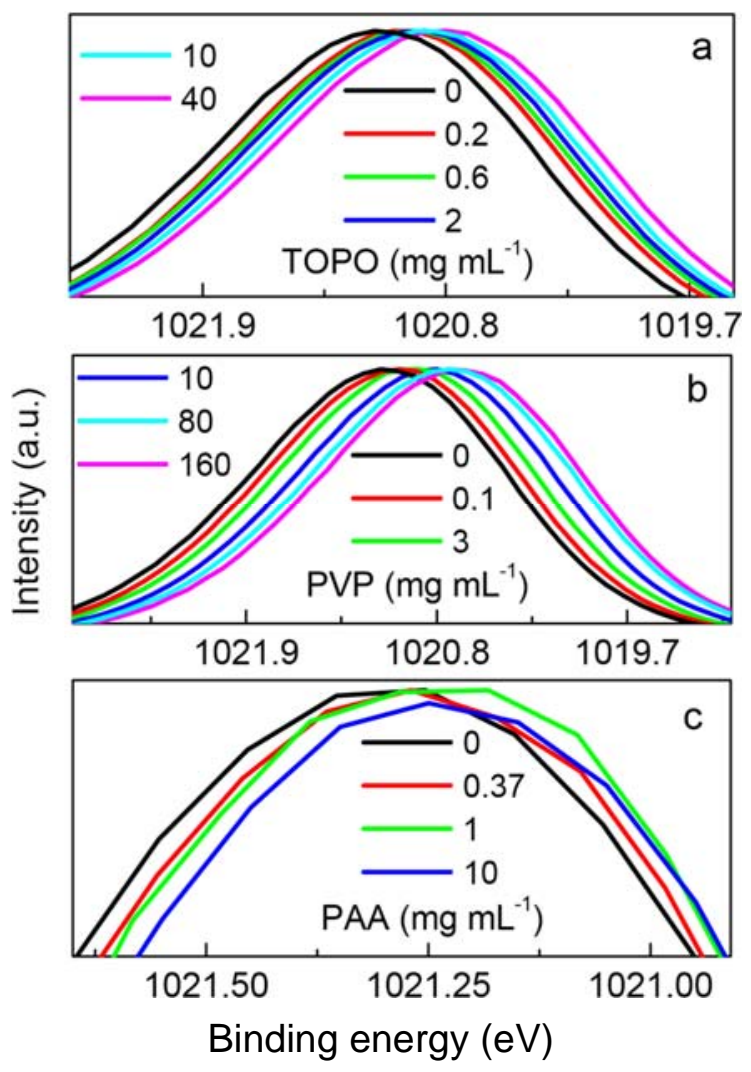

Figure 3, Zhang et al. 


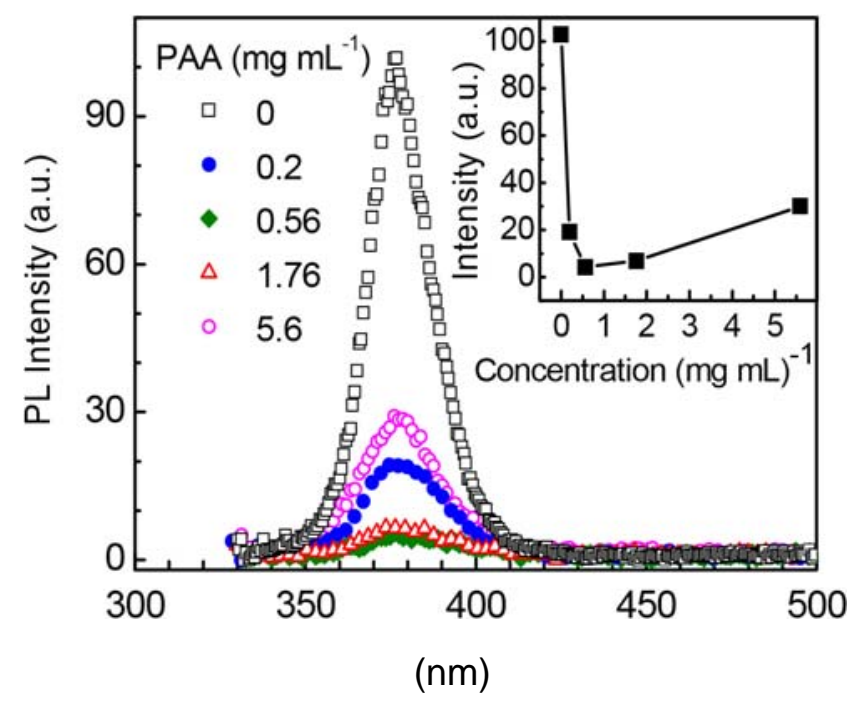

Figure 4, Zhang et al. 
This is an author-produced, peer-reviewed version of this article. The final, definitive version of this document can be found online at Physical Review B: Condensed Matter and Materials Physics, published by American Physical Society. Copyright restrictions may apply. DOI: 10.1103/PhysRevB.88.085437.
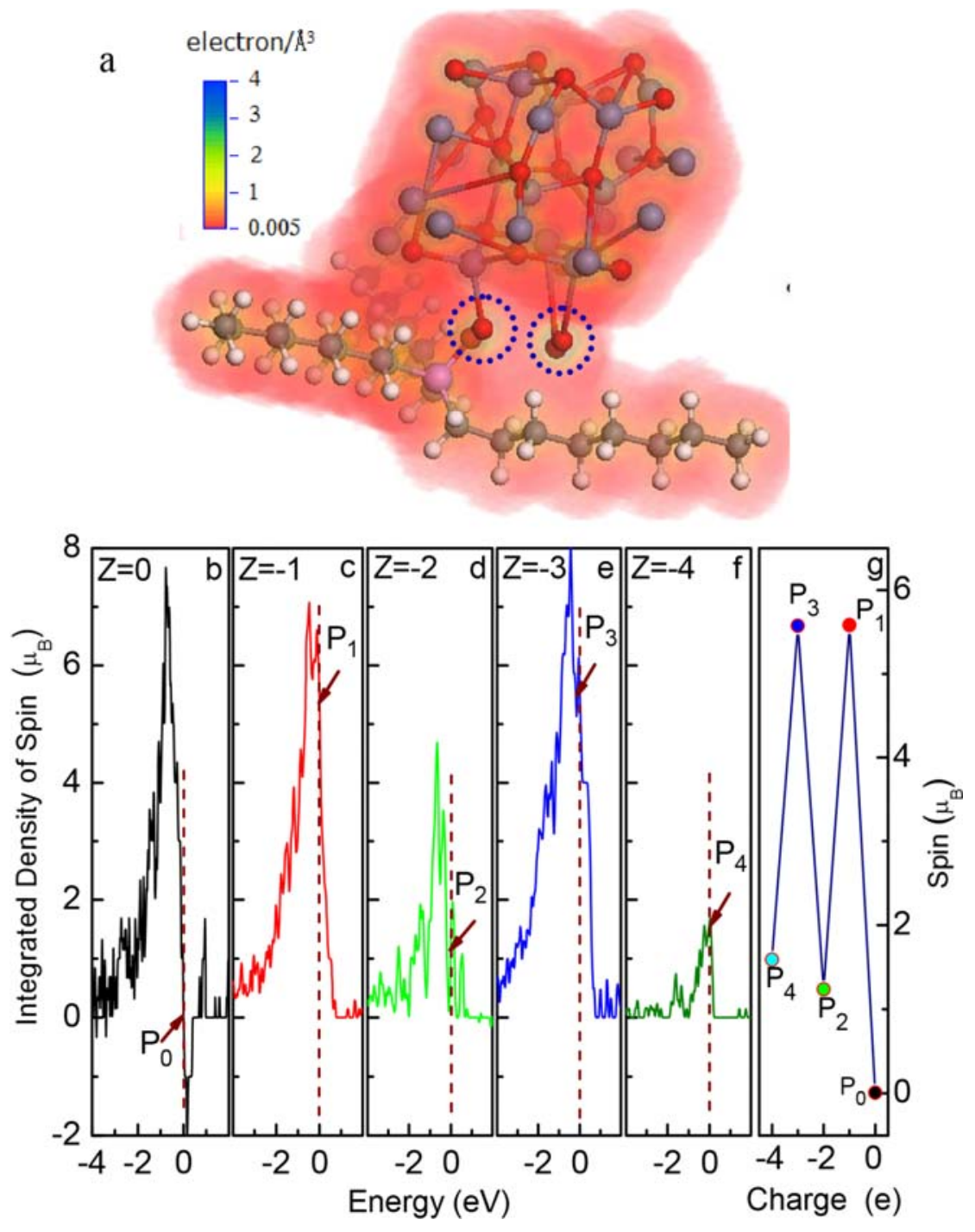